\title{
Quantification of earthquake diagnostic effects to assess low macroseismic intensities
}

\author{
Paola Sbarra $^{1}$ (D) Patrizia Tosi ${ }^{1} \cdot$ Valerio De Rubeis $^{1} \cdot$ Diego Sorrentino $^{1}$
}

Received: 9 April 2020 / Accepted: 21 August 2020 / Published online: 7 September 2020

(C) The Author(s) 2020

\begin{abstract}
A large amount of data about earthquake effects, supplied by citizens through a web-based questionnaire, enabled the analysis of the occurrence of many of the effects on humans and objects listed in macroseismic scales descriptions. Regarding the other diagnostic effects (rattling, moving, shifting, falling or overturning depending of the object type of doors, windows, china, glasses, small objects, pictures, vases, books, as well as frightened people and animal behaviour), data from more than 300,000 questionnaires about earthquakes felt in Italy from June 2007 to August 2017, were analysed by stacking them together as a function of hypocentral distance and magnitude. The comparison of the resulting percentages with the intensity prediction equation showed that almost all the chosen effects are good diagnostics for macroseismic intensity evaluation, as their percentages are well differentiated. We did not analyse the oscillations of hanging objects and liquids because the differences in effect attenuations, highlighted by the maps of the occurrence percentage, suggested to not consider them as diagnostic effect. This result allowed us to quantify the occurrence of each diagnostic effect for the intensity degrees from II to VI of the European macroseismic scale for the people who felt the earthquake. The application of the intensity assessment method to internet macroseismic data, based on the specifications herein proposed, should mitigate the problem of "not felt" undersampling in crowdsourced web data.
\end{abstract}

Keywords Diagnostic effects $\cdot$ EMS scale $\cdot$ Macroseismic questionnaire $\cdot$ Intensity estimation · Earthquake

\section{Introduction}

Macroseismic scales are based on experimental observations that are categorised to obtain the estimation of intensity levels of ground shaking. Objects and buildings can be considered as "instruments" in recording seismic effects, and even human response to ground shaking, when averaged over a large number of samples, can be a good indicator of the level of ground motion (Kayano 1990; Dengler and Dewey 1998). The descriptions of macroseismic scales detail an increase in the level of intensity of each diagnostic effect

Paola Sbarra

paola.sbarra@ingv.it

1 Istituto Nazionale di Geofisica e Vulcanologia, Via di Vigna Murata 605, 00143 Rome, Italy 
observed. As stated in the European macroseismic scale (EMS, Grünthal 1998), "The scale recognises the statistical nature of intensity, that is, that at any place a certain effect is likely to be observed in a proportion of cases only, and whether that proportion is small or large is itself something that tells one about the strength of the shaking." These trends, based mainly on reports from the field, but even on common sense, have been studied systematically in large samples of data only a few times (Brazee 1979; Vannucci et al. 2015) and the occurrence of each diagnostic for each intensity level has been poorly quantified using qualitative word such as few, many and most. Instead, directly observing the diagnostic effects of an earthquake without the conventional interpretation of an intensity scale should provide a record regardless of the adopted methodology (Musson 1992).

Nowadays, considering that crowdsourcing has gained more attention in several fields of interest, web-based macroseismic intensity questionnaires are used by many organisations (Radziminovich et al. 2014; Van Noten et al. 2017). The development of automated methods for determining intensity (Ringdal et al. 1978; Gasparini et al. 1992; Ferrari et al. 1995; Musson 2006; Mazet-Roux et al. 2010; Sbarra and Tosi 2010; Wald et al. 2012; Tosi et al. 2015; Vannucci et al. 2015; Goded et al. 2018) and the availability of large amounts of data have paved the way for the quantification of each diagnostic effect, thus guaranteeing a more objective assessment of macroseismic intensity.

The ease in obtaining information about effects has sharpened the focus of the scientific community on the low intensity, which are registered both far away from strong earthquakes and near the epicentre of small ones. The distinction among the lowest degrees of a macroseismic scale (from I to V) is mainly based on the percentage of people feeling the earthquake and on the occurrence of some specific diagnostic effects. The latter are not directly recorded, but indirectly reported by people who observed them. However, a problem connected with the use of online data, especially when they are mostly gathered from volunteers, is the scarcity of "not felt" reports, thus affecting the calculation of the "felt" percentage. It is then necessary to quantify the amount of these missed "not felt" responses (Boatwright and Phillips 2017). Despite the creation of the group of "registered users" in the INGV site, who are invited by e-mail to respond even if they have not felt the earthquake, the number of "not felt" responses received was underestimated by a factor of 10 (Tosi et al. 2015). Yet, this factor actually is highly variable depending on time, earthquake magnitude, geographical location, etc. For this reason, when assessing low intensities, it may be useful to consider each diagnostic effect as it has been reported by people who felt the earthquake and filled the questionnaire, specifying whether or not each effect was observed. Finally, by combining the different strength levels in only one class, each effect can be quantified as percentage of occurrence. This change of perspective to evaluate the macroseismic intensity is not straightforward, because one must consider that macroseismic effects may have different attenuation trends, as each effect is the response to a particular frequency range of ground shaking (Tosi et al. 2017). In fact, to determine reliable values of macroseismic intensity it is advisable, for each level of the scale, to consider only effects having the same trend of attenuation.

\section{Disagreements between diagnostics}

"Hai Sentito Il Terremoto" (which translates to "Did you feel the earthquake?" www. haisentitoilterremoto.it, hereafter HSIT, Tosi et al. 2007), managed by the Istituto Nazionale di Geofisica e Vulcanologia, collects responses concerning earthquakes felt in 
Italy since 1997 . The HSIT database contains the responses given to an online questionnaire (Sbarra et al. 2019) by both volunteers and approximately 27,000 registered users, who were invited, via email, immediately after the occurrence of an earthquake to give feedback. The questions focus on the diagnostic effects needed for the assessment of macroseismic intensity (Tosi et al. 2015; De Rubeis et al. 2019). Only one answer is possible for each multiple-choice question listing progressive strength levels, starting from the absence of the effect itself.

We mapped the occurrence of each diagnostic effect by calculating their percentage of observation in each municipality for the following three earthquakes, i.e. Emilia, 20 May $2012 \mathrm{Mw}$ 5.8, Norcia, 30 October $2016 \mathrm{Mw}$ 6.5, and Verona, ML 3.018 June 2013. The percentages obtained were the ratio between the number of answers describing the occurrence of the considered effect, combining the progressive strength levels in only one class (e.g. the effect on doors and windows combines the following levels "rattling", "opening or closing" and "slamming"), and the total number of answers including the absence of the effect itself. The percentages of all earthquake effects available on the HSIT site, calculated in the municipalities having more than 4 answers for each effect, are shown in Figs. 1, 2, and 3, respectively. The comparison of the thematic maps highlights the efficiency of each diagnostic in representing the shaking intensity. In fact, most of effects of the first two earthquakes (Figs. 1,2) showed a significant variation in the percentage of observation within a distance of $200 \mathrm{~km}$ from the epicentre. On the contrary, the maps of free-hanging objects (Figs. 1e, 2e) and liquids oscillation (Figs. 1i, 2i) reveal that the effect is almost equally present at all shown distances. These patterns match the results found by Tosi et al. (2017), who showed that, in the whole HSIT database, the mean percentage of occurrence of each effect, calculated as a function of hypocentral distance and magnitude, presents a specific attenuation trend identified by the ratio between magnitude and distance coefficients of the linear regression function. On the basis of this ratio, the diagnostic effects were separated by Tosi et al. (2017) into two groups. The first group accounted for most of the diagnostics, i.e. rattling, moving, shifting, falling or overturning depending of the object type (doors, windows, china, glasses, small objects, pictures, vases, books), frightened people and animal behaviour. The second group included oscillation of liquids and hanging objects. Tosi et al. (2017) suggested that the effects in the first group were the response to a $1 \mathrm{~Hz}$ ground motion, while the oscillations in the second group were the response to lower frequency ground motion. The frequency-dependent differences in attenuation are the reason why in places that should experience the same macroseismic intensity, i.e. far from medium-high earthquakes and near small ones, there are high percentages of oscillations in group 2 (Figs. 1e, i, 2e, i), or low percentages (Fig. 3e, i), depending on the magnitude. In detail, by comparing macroseismic fields (Fig. 4) and diagnostic maps (Figs. 1, 2, 3), it appears that for most municipalities having intensity III EMS of Emilia and Norcia earthquakes (Fig. 4a, b) the percentage of oscillations of both free-hanging objects and liquids is greater than $75 \%$ (Figs. 1e, i, 2e, i), while near the epicentre of the smaller event (Fig. 4c), where intensity is III EMS, the percentage of oscillations is on average $23 \%$ (Fig. 3e) for free-hanging objects and 35\% for liquids (Fig. 3i).

Thus effects involving oscillations could have different behaviours for high- and lowmagnitude earthquakes. As the scale should be equally valid for all earthquakes in the next section we analyse the HSIT database (De Rubeis et al. 2019; Sbarra et al. 2019) to quantify only the other effects showing similar attenuation behaviours, for each macroseismic intensity value. 


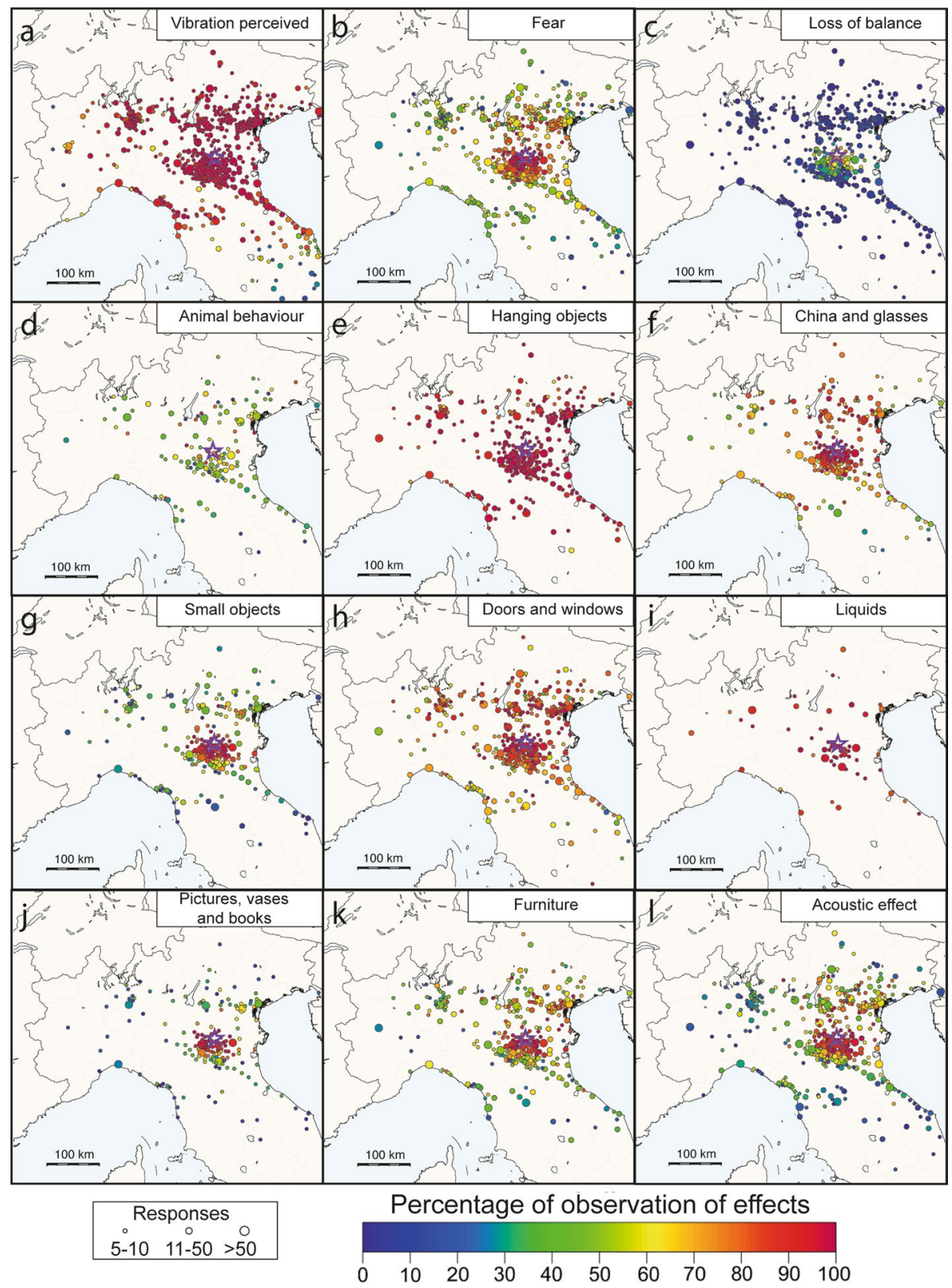

Fig. 1 Spatial distribution of observation percentages for each considered diagnostic effect for the Emilia earthquake, Mw 5.8, which occurred on 20 May 2012

Even if the data are scarce, we added the analysis of the loss of balance percentages (Figs. 1c, 2c, 3c) to the above-mentioned effects, because it is one of the diagnostic effects listed in the EMS characterising the medium-high degrees. 


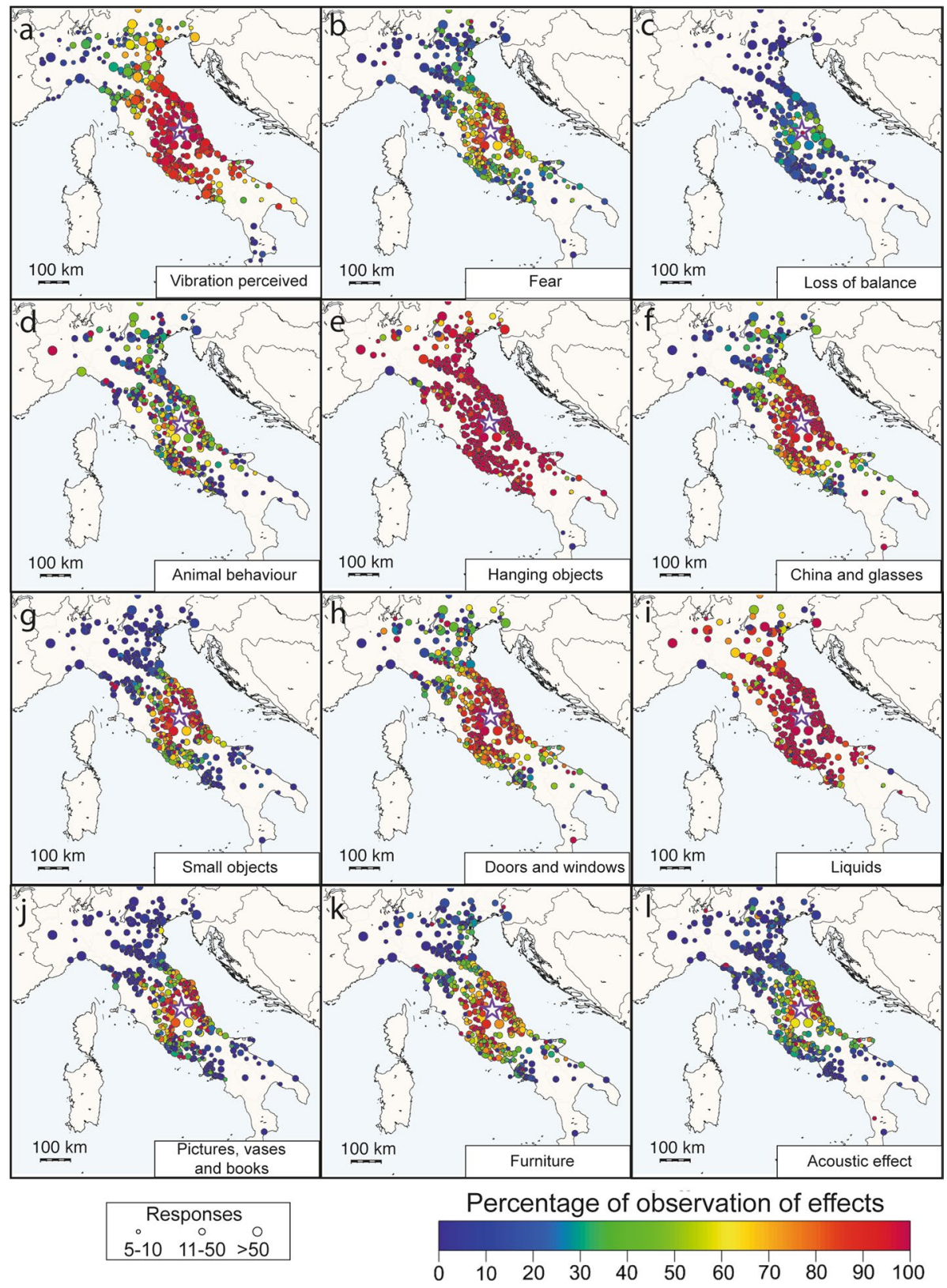

Fig. 2 Spatial distribution of observation percentages for each considered diagnostic effect for the Norcia earthquake, Mw 6.5, which occurred on 30 October 2016

\section{Quantification of the effects}

We analysed 315,991 indoor "felt" questionnaires concerning 11,253 earthquakes that occurred from June 2007 to August 2017 in Italy or in the neighbouring countries (Fig. 5). 


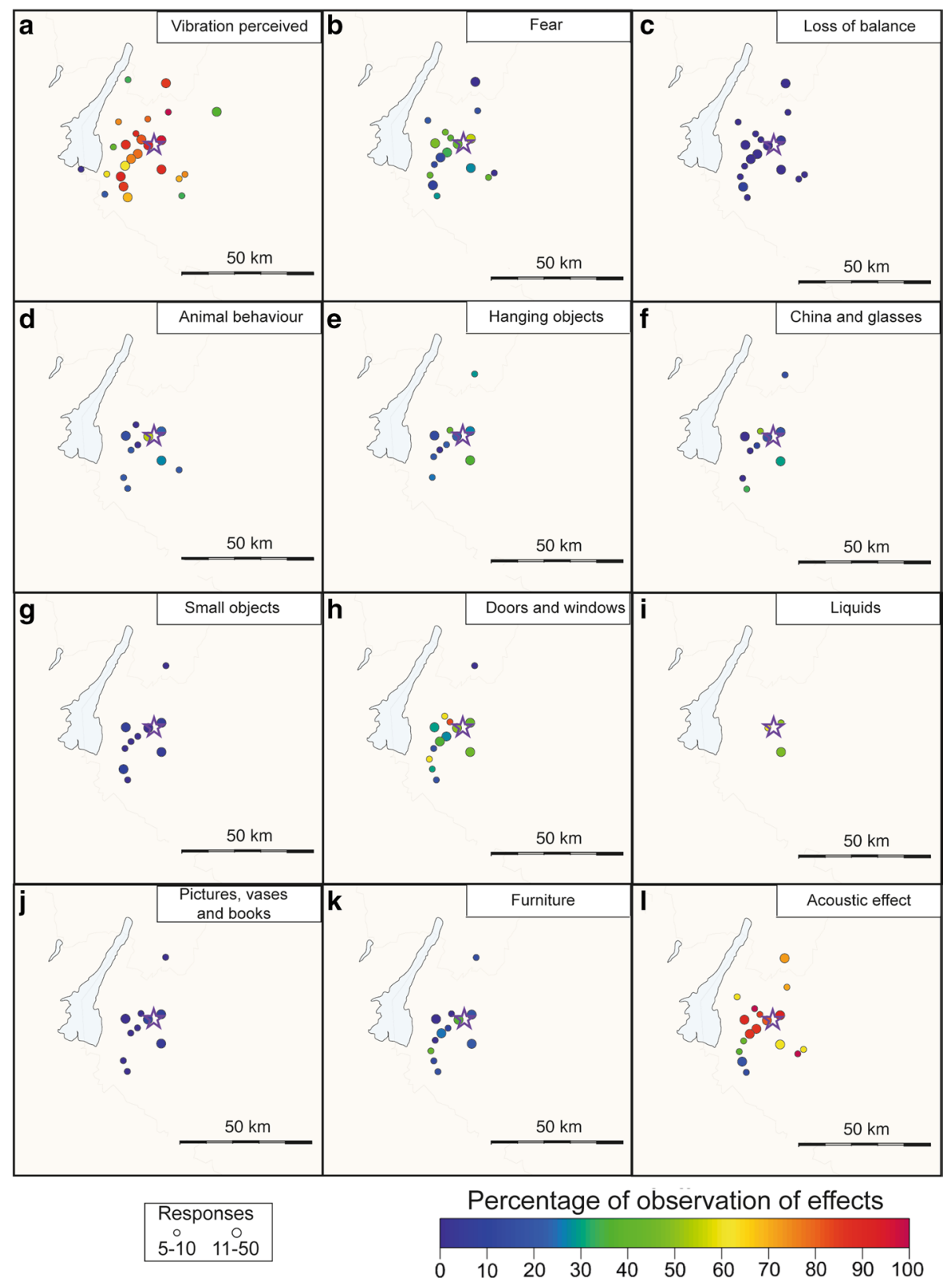

Fig. 3 Spatial distribution of observation percentages for each considered diagnostic effect for the Verona earthquake, ML 3.0, which occurred on 18 June 2013

The data set consists of events with a magnitude (ML) range from 2 to 6.1, and depths shallower than $35 \mathrm{~km}$, that received more than 3 questionnaires. Some of the aftershocks, or other events occurring in a short space of time, were excluded because of the risk of people confusing them with the mainshock events (Tosi et al. 2017). 
Fig. 4 EMS macroseismic intensity maps assessed with HSIT online questionnaire data. The purple stars indicate the epicentres. a 20 May 2012, Mw 5.8, lat. $11.23^{\circ} \mathrm{N}$ long. $44.89^{\circ}$ E. b 30 October 2016, Mw 6.5, lat. $13.11^{\circ} \mathrm{N}$ long. $42.84^{\circ} \mathrm{E}$. c 18 June 2013, ML 3.0, lat. $10.98^{\circ} \mathrm{N}$ long. $45.54^{\circ} \mathrm{E}$

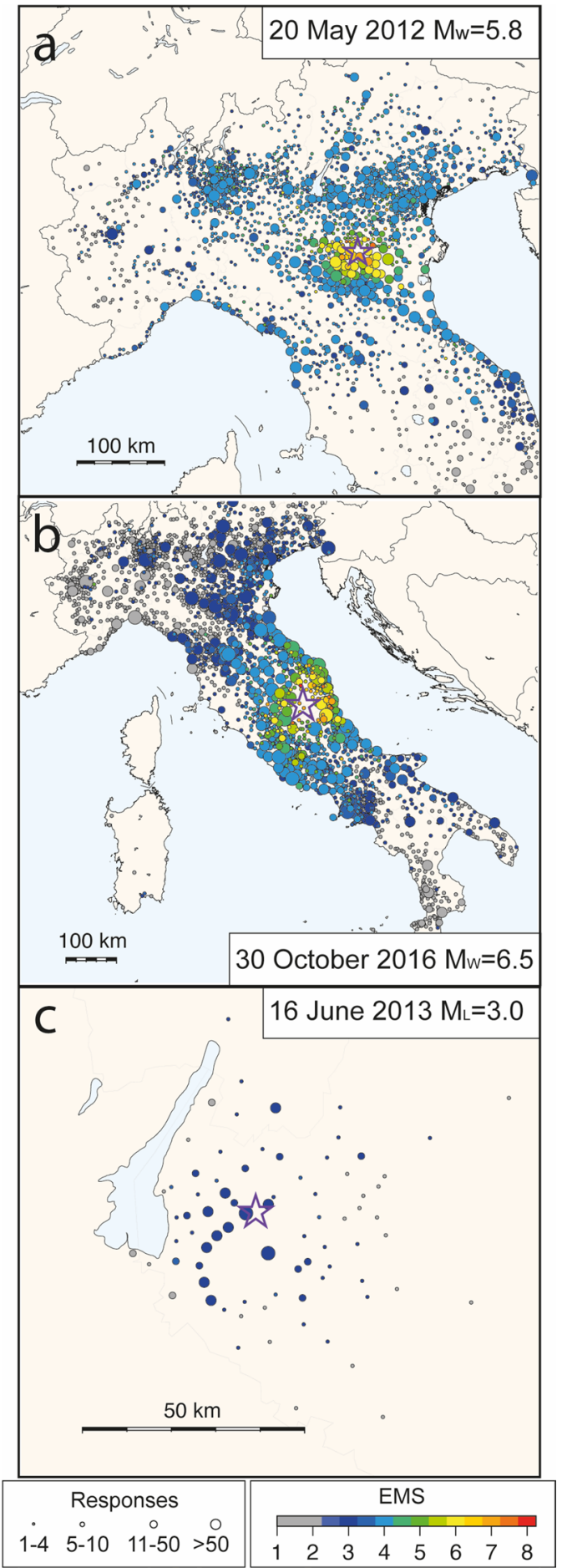




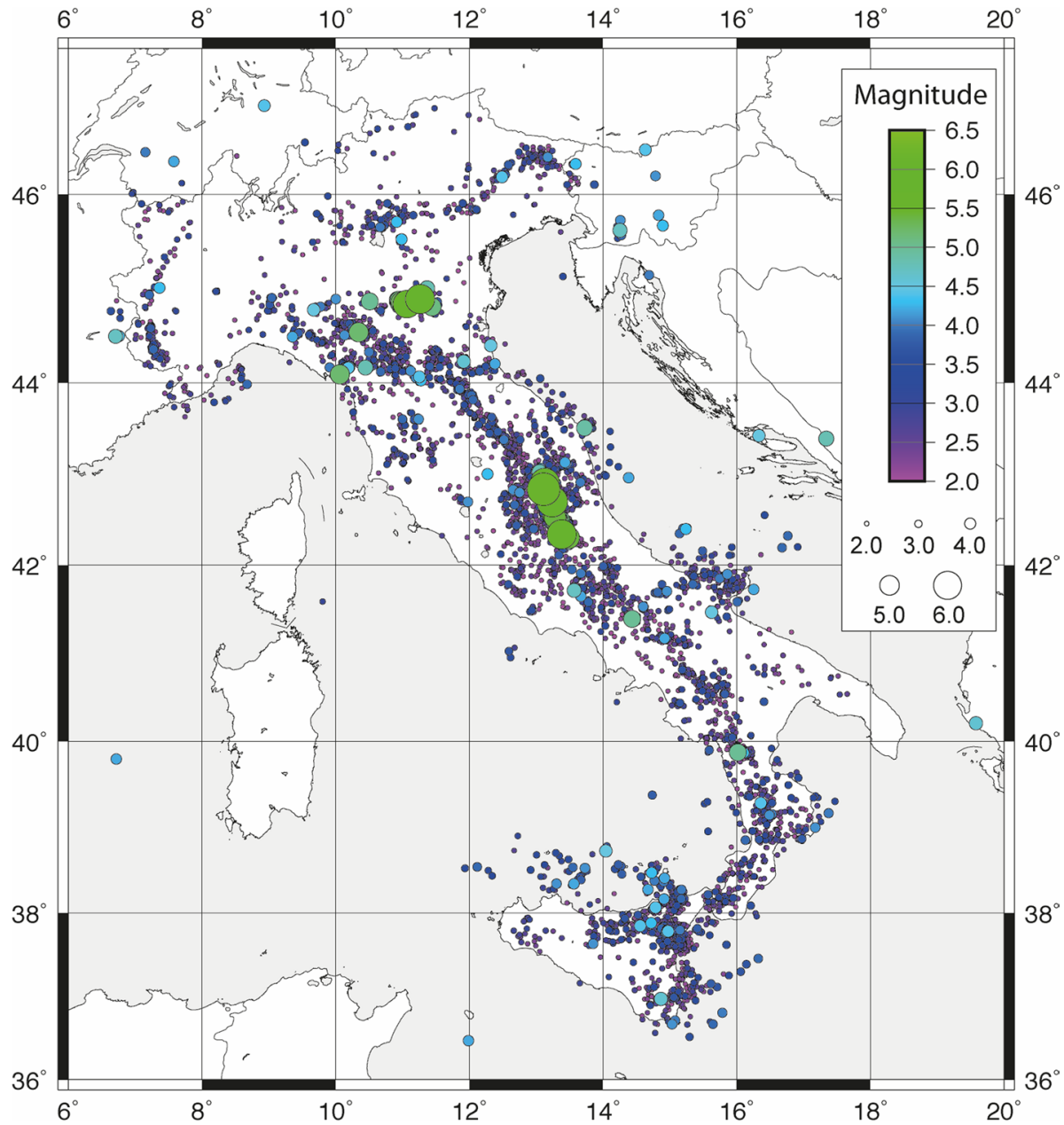

Fig. 5 Map of 11,253 earthquakes analysed in this study, which occurred from June 2007 to August 2017

For each effect, the answer "unable to say", a different option from the absence of the effect itself, was selected in about $45 \%$ of responses, with an almost homogeneous distribution as a function of distance and magnitude. Our data show that this answer was chosen by only $10 \%$ of people for earthquakes having a magnitude greater than 5.8 and hypocentral distances less than $30 \mathrm{~km}$, showing that with high-intensity earthquakes citizens fill the macroseismic questionnaire with greater care, by responding to almost all questions.

The more frequently answered questions concerned the effects on people (fear 304,170 answers; 92\%) and on doors or windows (233,765 answers; 71\%), while questions about the behaviour of animals (105,032 answers; 32\%) were answered less frequently (Table 1).

To quantify the diagnostics, we calculated the percentage of occurrence of each effect, stacking data of all selected earthquakes, as a function of hypocentral distance and magnitude, following Tosi et al. (2017). Each magnitude-distance window included at least 3 answers and more than 1000 answers at most, with a median of about 100 values. The resulting percentages (Fig. 6), as expected, show similar trends among the 
Table 1 Number of analysed macroseismic questionnaires, regarding 11,253 earthquakes, with magnitude range from 2 to 6.1 (ML), felt in Italy from June 2007 to August 2017

\begin{tabular}{lclc}
\hline Diagnostic effect & $\begin{array}{l}\text { Number of people } \\
\text { who answered } \\
\text { "yes" }\end{array}$ & $\begin{array}{l}\text { Number of people } \\
\text { who answered } \\
\text { "no" }\end{array}$ & $\begin{array}{l}\text { Number of people who } \\
\text { answered "unable to say" }\end{array}$ \\
\hline Fear (people is frightened) & 118,165 & 186,005 & 27,352 \\
Loss of balance & 10,561 & 280,787 & 40,174 \\
Animal behaviour (become uneasy) & 29,821 & 75,211 & 226,490 \\
China and glasses (rattle or clatter & 72,168 & 99,662 & 159,692 \\
$\quad$ together) & 34,327 & 141,613 & 155,582 \\
Small objects (shift or fall) & 126,248 & 107,517 & 97,757 \\
Doors and windows (rattle, open or & & & 186,803 \\
$\quad$ shut) & 22,887 & 121,832 & 125,194 \\
$\quad$ Pictures, vases and books (shift or & & 144,620 & \\
Furniture (shift or overturn) & 61,708 & &
\end{tabular}

The diagnostic effect is indicated inside the brackets as reported in the scale descriptions

effects. Such behaviour is in agreement with results by Tosi et al. (2017) although data set in that work was limited to October 2015 and the number of analysed questionnaires was $20 \%$ lower than the data set here examined.

All the effects shown in Fig. 6 are used in the macroseismic scales as diagnostics, and thus reasonably their attenuation as a function of magnitude and distance closely follows macroseismic intensity, which by definition is based on the whole set of effects involved. To compare the attenuations of each single effect with that of EMS macroseismic intensity (I), the isolines of a simple Intensity Prediction Equation (IPE), were also drawn in Fig. 6 (black diagonal lines). These isolines were obtained using the method of Tosi et al. (2015) on the HSIT intensity data,

$$
I=(-2.55 \pm 0.03) \log _{10} D+(1.08 \pm 0.01) M_{L}+2.43 \pm 0.04
$$

where $D$ is the hypocentral distance. Figure 6 shows that the isolines of the IPE, for each single effect, border magnitude-distance windows having similar percentages of the occurrence for the effect itself, and thus, that the attenuation of each effect has the same trend as the EMS IPE. As an example, from Fig. 6, let us consider the percentages of frightened people included between III and IV EMS. This interval includes percentages in the 40-60\% range, in agreement with all the distance-magnitudes windows. In other words, there is a good correspondence between percentage and intensity intervals. This correspondence allows the estimation of a percentage of people who observed each effect for every EMS intensity value. The average of percentages corresponding to windows having the same EMS intensity predicted by Eq. 1 is shown in Fig. 7.

To exemplify the results of Fig. 7, we summarised in Table 2 the percentages of the effects on objects and humans which should be operatively applied to improve the estimation of EMS intensities from II to VI. We also inserted in Table 2 the quantification of the vibration perceived, already published by Sbarra et al. (2014), in order to include all the results on transient effects. As specified, it is important to point out that the percentages were calculated among those who felt the earthquake. 

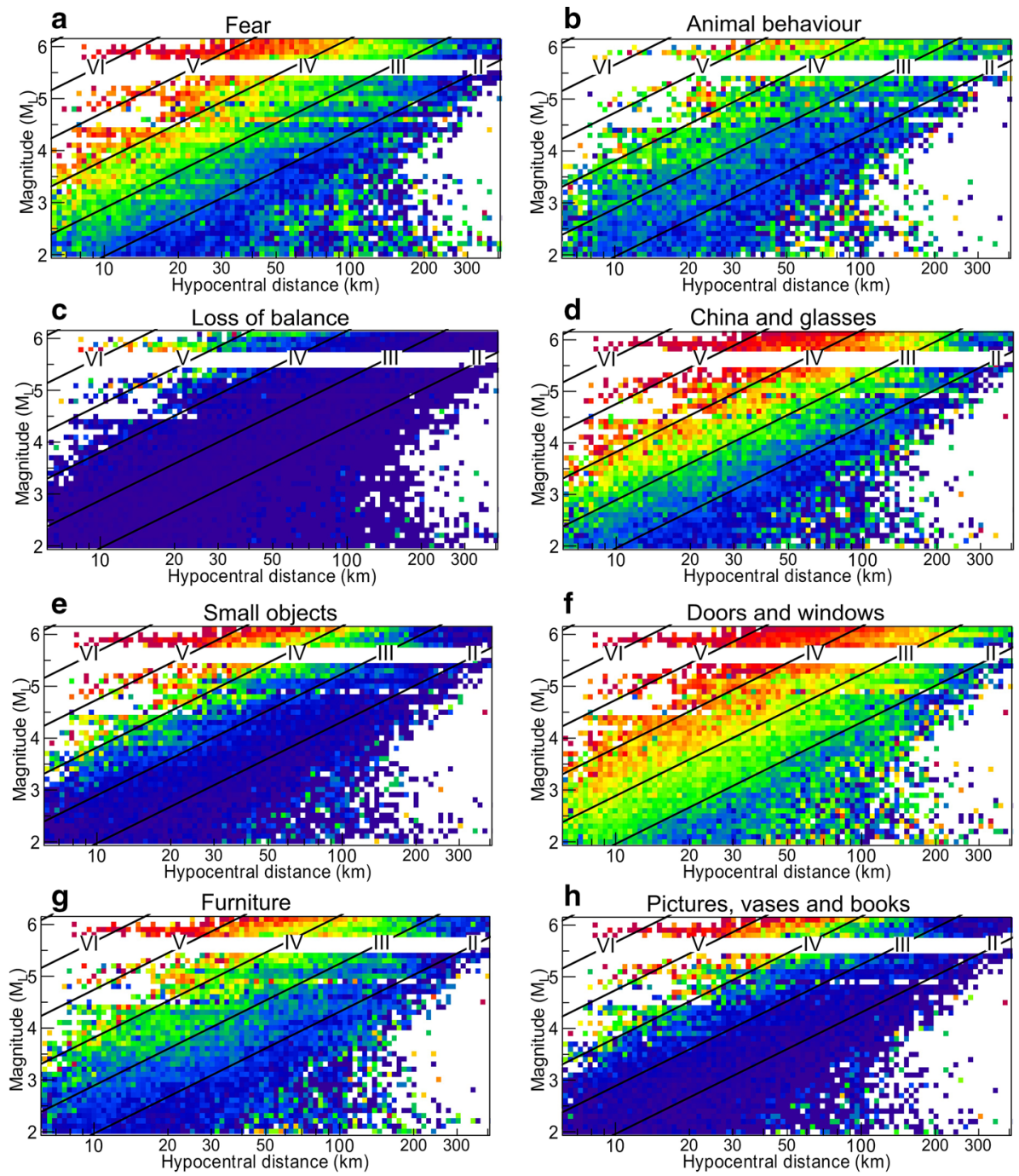

Percentage of observation of effects

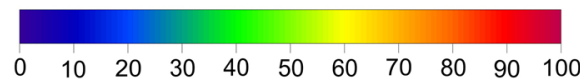

Fig. 6 Distribution in percentages of observation colour-coded as a function of magnitude and hypocentral distance for each diagnostic effect analysed. The black diagonal lines represent intensities in EMS scale calculated using Eq. 1. The percentages were obtained summing up the data pertaining to all earthquakes of a defined magnitude (with a precision $\Delta m=0.1$ ), within a logarithmically increasing range of hypocentral distance (window length $=0.02 \log _{10} \mathrm{D}$, where $\mathrm{D}$ is the hypocentral distance expressed in $\mathrm{km}$ )

We include in this graph also the diagnostic of the movement of pictures, vases and books although it is not mentioned in the EMS scale. This diagnostic is similar to the movement of small objects; in fact, they show a similar trend (Fig. 7), but it is possible to observe some differences in the percentage of occurrence of each one. Therefore, we 
Fig. 7 Percentage of people who observed each diagnostic effect (this study) versus the EMS intensity. A third-degree polynomial regression line connects the points in order to highlight the trend

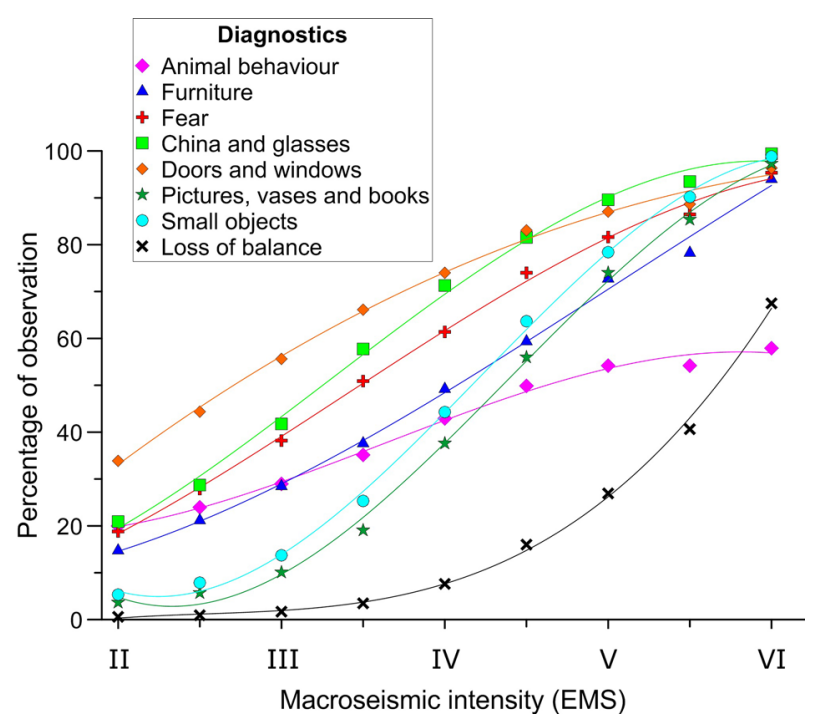

believe that these two diagnostics must remain separate and we propose adding the movement of pictures, vases and books to the EMS scale.

\section{Discussion}

The results of the HSIT data analysis show that most effects follow the attenuation trend of Eq. 1. This indicates that they are good diagnostics for intensity estimation and confirms the reliability of citizens' reports. Here, to focus on a smaller number of variables, we did not consider the specific location (floor and building height) of the observers (Sbarra et al. 2012, 2015; Van Noten et al. 2017; Schlupp et al. 2018). However, the floors higher than the fifth account for only $5 \%$ of our database. Besides, the potential effects of other major variables, such as site geology and focal mechanism, were neglected in this analysis, assuming that these variables were balanced and averaged due to the heterogeneity of the data contained in the large HSIT data set, which covers the entire Italian territory and presents a moderately large magnitude range.

The analysis of effects attenuation highlighted the different behaviour of the oscillations of hanging objects and liquids with respect to other effects (Figs. 1, 2, 3). In fact, the percentages of observed oscillation of hanging objects do not follow specific intensity levels. The same behaviour was observed for the oscillation of liquids, suggesting that the oscillations in general, probably even of bells, can introduce a noise component in intensity assessment. In particular, considering that the oscillation of chandeliers is a frequently observed effect, and that this effect is often drawn on thumbnails depicting earthquake intensity (Sira 2015; Bossu et al. 2017; Goded et al. 2017), there can be an anomalous extension of III degree at the expense of II in the case of high-magnitude, and the opposite in the case of low-magnitude events (for these reasons, Le Bureau Central Sismologique Français followed our suggestions and modified the last version of the miniatures, presented after the on-line questionnaire, and removed the hanging objects from the pictures, https://www.franceseisme.fr/formulaire/index.php?IdSei=0). Actually, the swinging of 


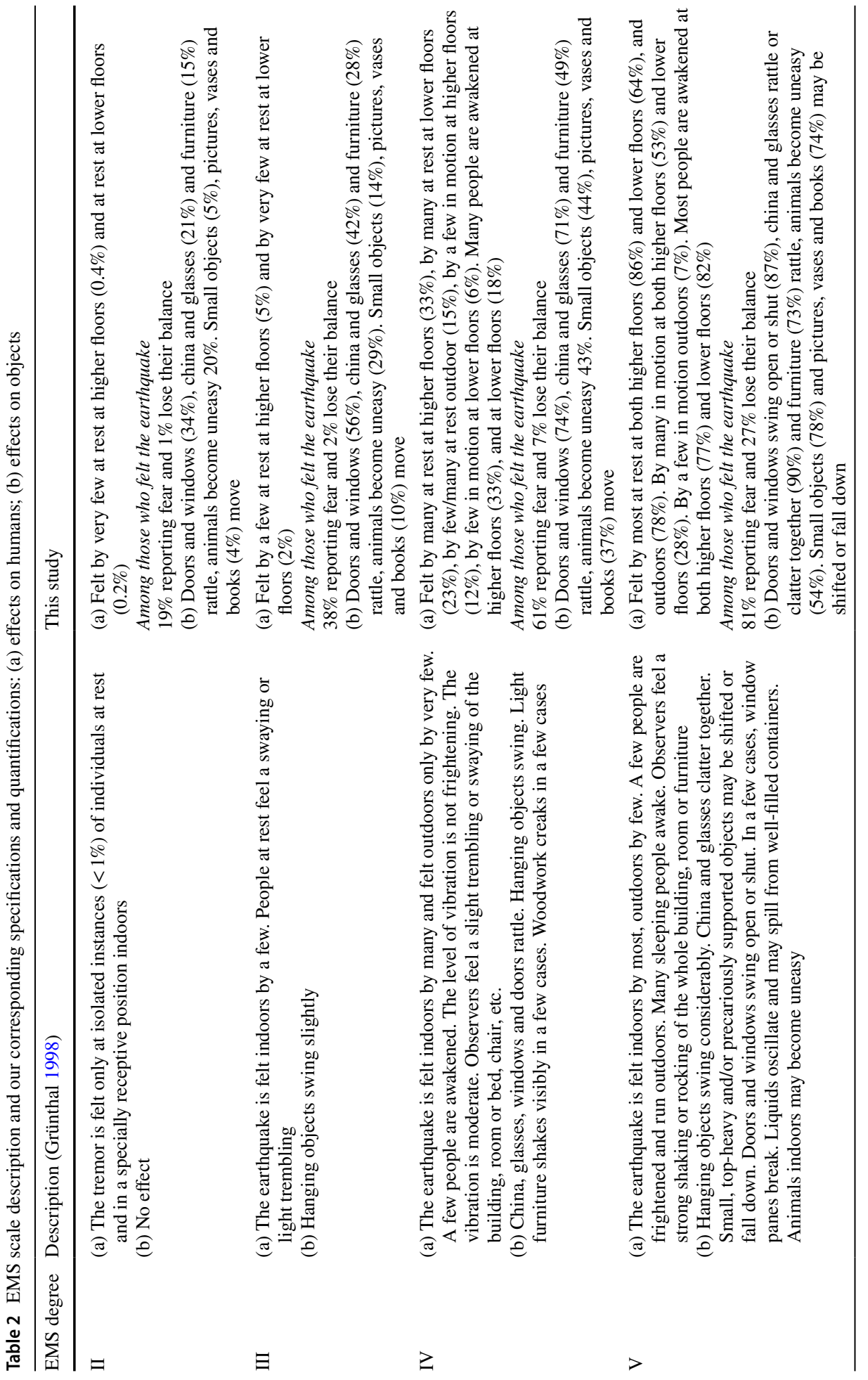




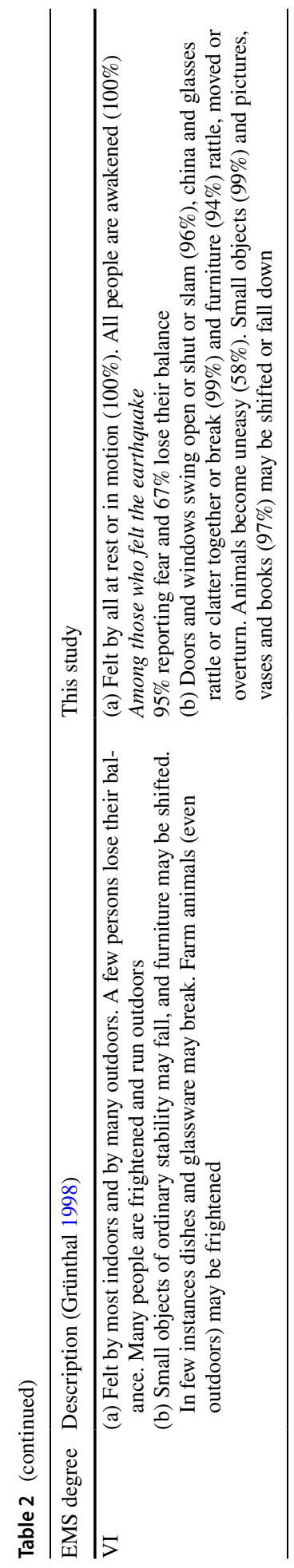


hanging objects seems to be a controversial diagnostic. Its claimed intensity level varies significantly in different macroseismic scales, even though intensities lower than VI in the Mercalli-Cancani-Sieberg scale (MCS, Sieberg 1930), the EMS, Mercalli Modified Intensity (MMI, Wood and Neumann 1931) and the Medvedev-Sponheuer-Karnik scale (MSK, Medvedev et al. 1965) should be very similar to each other (Molin 1995; Musson et al. 2010). For example, the EMS scale defines three levels of swinging (slightly at III EMS, moderately at IV EMS and considerably at V EMS, see Table 2). The MMI scale reports in II "sometimes may swing", in III "may swing slightly", in IV "swung in numerous instances", in V "swing generally or considerably". The newer version of MMI (Musson and Cecić 2012) reports the same descriptions, omitting hanging objects in degree V. MCS and MSK scales report only one level (swinging of chandeliers at degree V). Finally, the scale adopted by the Japan Meteorological Agency (JMA 2019; Musson and Cecić 2012), considering that 2 and 4 JMA respectively correspond to IV and V EMS (Musson et al. 2010; Musson and Cecić 2012), quotes "swing slightly" in intensity 2, "swing considerably" in 4 and "swing violently" in 5-Lower. In summary, this diagnostic of lowintensity degrees is discordant in different scales. Moreover, for medium-high magnitudes, it is observed up to very long distances; instead, it does not characterise low-magnitude events. As an example, the slight swinging of hanging objects is almost never observed in Britain (Musson 1991) where seismicity is characterised by low magnitudes (Grünthal 1989). This problem was already mentioned in the specific notes of the EMS scale guidelines (Grünthal 1998). For all these reasons we suggest to critically revise the diagnostic of hanging object oscillation and/or to rephrase the correspondence question inside the macroseismic questionnaire.

Similar considerations apply to the oscillation of liquids that are currently contemplated, in many macroseismic scales. We here list some scale descriptions. MMI (Wood and Neumann 1931) intensity I "liquids may sway very slowly", II "liquids may swing", IV "disturbed liquids in open vessels slightly", V "spilled liquids", VI "liquid set in strong motion"; EMS intensity V "liquids oscillate and may spill", VII "water splashes from containers, tanks and pools"; MCS scale intensity IV "oscillating slightly", V "water may spill from filled container", VI "moving strongly"; MSK intensity V "liquids oscillate and may spill". Therefore, in all these scales, the presence of this diagnostic could generate a noise component in the intensity assessment. Thus, we discourage the use of this diagnostic as suggested by Stover and Coffman (1993).

Regarding the diagnostics with the attenuation behaviour similar to that of intensity (Fig. 7), the movement of doors and windows is the most perceived effect at the lowest EMS intensities (Table 2). Regarding IV EMS, in the scale of perception the following effects are observed (the mean percentage in brackets): movement of doors and windows (74\%), movement of china and glassware (71\%), fear (61\%), movement of furniture (49\%), animal behaviour (43\%), movement of small objects (44\%), movement of pictures, vases and books (38\%), and loss of balance (8\%). All the percentages increase quite steeply towards VI EMS, except for animal behaviour that never exceeded the value of $60 \%$. Thus, this diagnostic, considered in the description of V EMS, turned out to be a poor quality marker, as its percentages for different intensities change slightly. It is worth noting that this effect could be more difficult to evaluate, as it relies on the ability of the compiler to interpret the reaction of animals. Recently, Woith et al. (2018) highlighted the difficulties in collecting and analysing this kind of data, in a review of 44 publications and 729 reports on abnormal animal behaviour in connection with 160 earthquakes.

In Fig. 7, some diagnostic effects show significant percentages of occurrence, among those who felt the earthquake, even in II EMS (Table 2). That is, $34 \%$ for door and window 
rattling, $21 \%$ for rattling of china and glasses, $20 \%$ for animal behaviour is $20 \%, 19 \%$ for people reporting fear, and $15 \%$ for the movement of furniture. Thus, it is possible to assess II and III EMS, in web-based surveys, even on the basis of these diagnostics, which are usually neglected in the lowest intensities. The percentage of occurrence of each effect could then be used, especially for Internet surveys, when sufficient data are available, to recognise low and medium intensities (from II to VI, Fig. 7). Moreover, the use of percentages based only on questionnaires filled by citizens who felt the earthquake, reduces the problem of the underestimation of not felt reports raised by Boatwright and Phillips (2017).

Percentage values shown in Fig. 6 and Table 2 are based on the expected intensities calculated with Eq. 1, but we want to emphasise that even using the experimental EMS intensity values assessed for each municipality by the HSIT system (Tosi et al. 2015) we obtained similar values, thus confirming the robustness of results. We preferred the first approach, to avoid potentially wrong local intensities resulting from the incoherence of diagnostics in low degrees.

\section{Conclusions}

This paper is focused on quantification of the occurrence of effects on humans and observed effects objects to reduce the subjective component in macroseismic intensity assessment. In fact, many authors (Davison 1921; Ferrari and Guidoboni 2000; Musson et al. 2010; Musson and Cecić 2012; Vannucci et al. 2015) admit that one of the difficulties in assessing macroseismic intensity is the rough quantification of each diagnostic effect.

On the basis of the analysis of a large crowdsourced macroseismic data pool, it was verified that most of the diagnostic effects mentioned in the macroseismic scales are good markers for ground shaking intensity differentiation (Figs. 6, 7). Fear, a parameter that could be easily biased by subjective emotional states, has also proven to be a good marker of macroseismic intensity, whereas animal behaviour had a low resolution for distinguishing among medium intensities (from IV to VI EMS).

On the other hand, the oscillations of hanging objects and liquids were more related to magnitude variations than other effects. Consequently, following the results of this article, we suggest omitting or at least critically revising the oscillation of hanging objects and liquids within diagnostics, to assure a magnitude-independent scale. We hope that our results can be a starting point for a discussion and comparative research in other countries, to propose, in case, a change of the EMS scale. As mentioned by Grünthal (1998) in the guidelines and background material of the latest version of the EMS scale, "future applications or future needs might be the basis for further improvements" of a macroseismic scale. The results presented here (summarised in Table 2) suggest that the estimation of II and III intensity degrees can be improved, by considering the percentage of occurrence of some diagnostics (movement of doors, windows, china, glasses, and fear) reported by people who felt the earthquake. The quantification, in terms of percentage of observation of each diagnostic effect on objects and humans, could allow a more objective intensity assessment for EMS intensities from II to VI.

Acknowledgements This work would not have been possible without the efforts of so many people in Italy who have filled in the HSIT macroseismic questionnaires, providing us with valuable data. We are grateful to Pierfrancesco Burrato and Roberto Vallone for helpful advice. We acknowledge the financial support of the INGV-DPC 2019-2021 agreement (Allegato A, WP7). 
Funding Open access funding provided by Istituto Nazionale di Geofisica e Vulcanologia within the CRUICARE Agreement.

Open Access This article is licensed under a Creative Commons Attribution 4.0 International License, which permits use, sharing, adaptation, distribution and reproduction in any medium or format, as long as you give appropriate credit to the original author(s) and the source, provide a link to the Creative Commons licence, and indicate if changes were made. The images or other third party material in this article are included in the article's Creative Commons licence, unless indicated otherwise in a credit line to the material. If material is not included in the article's Creative Commons licence and your intended use is not permitted by statutory regulation or exceeds the permitted use, you will need to obtain permission directly from the copyright holder. To view a copy of this licence, visit http://creativecommons.org/licenses/by/4.0/.

\section{References}

Boatwright J, Phillips E (2017) Exploiting the demographics of "Did you feel it?" Responses to estimate the felt area of moderate earthquakes in California. Seismol Res Lett 88:335-341. https://doi. org/10.1785/0220160041

Bossu R, Landès M, Roussel F et al (2017) Thumbnail-based questionnaires for the rapid and efficient collection of macroseismic data from global earthquakes. Seismol Res Lett 88:72-81. https://doi. org/10.1785/0220160120

Brazee RJ (1979) Reevaluation of modified Mercalli intensity scale for earthquakes using distance as determinant. Bull Seismol Soc Am 69:911-924

Davison C (1921) On scales of seismic intensity and on the construction and use of isoseismal lines. Bull Seismol Soc Am 11:95-130

Dengler LA, Dewey JW (1998) An intensity survey of households affected by the Northridge, California, Earthquake of 17 January 1994. Bull Seismol Soc Am 88:441-462

De Rubeis V, Sbarra P, Tosi P, Sorrentino D (2019) Hai Sentito Il Terremoto (HSIT)—macroseismic intensity database 2007-2018, version 1. https://doi.org/10.13127/HSIT/I.1

Ferrari G, Gasperini P, Guidoboni E (1995) Macroseismic intensity evaluation with the "Fuzzy sets logic". Ann Geofis 38:811-826

Ferrari G, Guidoboni E (2000) Seismic scenarios and assessment of intensity: some criteria for the use of the MCS scale. Ann Geofis 43:707-720

Gasparini C, De Rubeis V, Tertulliani A (1992) A method for the analysis of macroseismic questionnaires. Nat Hazards 5:169-177. https://doi.org/10.1007/bf00127004

Goded T, Horspool N, Gerstenberger M et al (2017) A comparison between GeoNet's "Felt RAPID" and "Felt detailed" online questionnaires. In: Proceedings of the New Zealand society of earthquake engineering technical conference, Wellington (New Zealand), April 2017

Goded T, Horspool N, Canessa S et al (2018) New macroseismic intensity assessment method for New Zealand web questionnaires. Seismol Res Lett 89:640-652. https://doi.org/10.1785/0220170163

Grünthal G (1989) Thoughts and proposals for the updating of the MSK intensity scale. Central Institute for the Physics of the Earth, Potsdam, pp 1-63

Grünthal G (1998) European macroseismic scale 1998 (EMS-98). Cahiers du Centre Européen de Géodynamique et de Séismologie Luxembourg 15:1-99

Japan Meteorological Agency (JMA) (2019) Summary of tables explaining the JMA seismic intensity scale. https://www.jma.go.jp/jma/kishou/know/shindo/kaisetsu.html. Last Accessed Sept 2019

Kayano I (1990) Distribution of various effects and damages caused by earthquakes and of seismic intensities on the basis of questionnaire surveys: a newly developed group survey method. Bull Earthq Res Inst Univ Tokyo 65:463-519

Mazet-Roux G, Bossu R, Carreño E et al (2010) EMSC real time earthquake information services. In: European-Mediterranean Seismological Centre (EMSC) report, 31 p. https://www.emsc-csem.org/ Files/news/EMSC/EMSC_RTEI_services.pdf. Last Accessed Sept 2019

Medvedev SV, Sponheuer W, Karnik V (1965) Seismic intensity scale version MSK 1964. Academy of Sciences of the USSR, Soviet Geophysical Committee, Moscow

Molin D (1995) Considerations on the assessment of macroseismic intensity. Ann Geofis 38:805-810

Musson RMW (1991) The use of the MSK intensity scale in the study of British earthquakes. In: Kozák J (ed) Proc 3rd Int Sym on Hist Eqs in Europe, Liblice, April 1990. Geoph. Inst. Czech. Acad. Sci., Prague 
Musson RMW (1992) Single diagnostic analysis of earthquake effects. In: Cecić I (ed) Proceedings 2nd AB workshop on macroseismic methods, Poljče, Yugoslavia, 15-18 Oct 1990, Seismological Survey of Slovenia, Ljubljana (Also as BGS Global Seismology Report No WL/91/14)

Musson RMW (2006) Automatic assessment of EMS-98 intensities. British Geological Survey technical report, IR/06/048.

Musson RMW, Cecić I (2012) Intensity and intensity scales. New manual of seismological observatory practice (NMSOP-2). IASPEI, GFZ German Research Centre for Geosciences, Potsdam, 2012. https://doi.org/10.2312/GFZ.NMSOP-2

Musson RMW, Grünthal G, Stucchi M (2010) The comparison of macroseismic intensity scales. J Seismol 14:413-428. https://doi.org/10.1007/s10950-009-9172-0

Radziminovich YB, Khritova MA, Gileva NA (2014) Modern methods for acquisition of macroseismic data and their possible uses for eastern Siberia. J Volcanol Seismol 8:375-389

Ringdal F, Husebye ES, Sandvin OA, Christoffersson A (1978) Statistical test theory in the analysis of macroseismic questionnaires. Tectonophysics 49:161-170

Sbarra P, Tosi P, De Rubeis V (2010) Web-based macroseismic survey in Italy: method validation and results. Nat Hazards 54:563-581. https://doi.org/10.1007/s11069-009-9488-7

Sbarra P, Tosi P, De Rubeis V, Rovelli A (2012) Influence of observation floor and building height on macroseismic intensity. Seismol Res Lett 83:261-266. https://doi.org/10.1785/gssrl.83.2.261

Sbarra P, Tosi P, De Rubeis V (2014) How observer conditions impact earthquake perception. Seismol Res Lett 85:306-313. https://doi.org/10.1785/0220130080

Sbarra P, Fodarella A, Tosi P, De Rubeis V, Rovelli A (2015) Difference in shaking intensity between short and tall buildings: known and new findings. Bull Seismol Soc Am 105:1803-1809. https://doi. org/10.1785/0120140341

Sbarra P, Tosi P, De Rubeis V, Sorrentino D (2019) Hai Sentito Il Terremoto (HSIT) -macroseismic questionnaire database 2007-2018, version 1. https://doi.org/10.13127/HSIT/Q.1

Schlupp A, Sira C, Dumetz C, Masson R (2018) Quantification of the increase in shaking with the floor level based on macroseismic data collected by the BCSF-RENASS in France. In: 36th general assembly of the European seismological commission, 2-7 Sept 2018, Valletta-Malta

Sieberg A (1930) Scala MCS (Mercalli-Cancani-Sieberg). Geologie der Erdbeben Handbuch der Geophysik 2:552-555

Sira C (2015) Macroseismic Intervention Group: the necessary field observation. In: Perspectives on European earthquake engineering and seismology. Springer, Berlin, pp 395-408

Stover CW, Coffman JL (1993) Seismicity of the United States, 1568-1989 (revised). US Government Printing Office

Tosi P, De Rubeis V, Sbarra P, Sorrentino D (2007) Hai Sentito Il Terremoto (HSIT) https://doi. org/10.13127/HSIT

Tosi P, Sbarra P, De Rubeis V, Ferrari C (2015) Macroseismic intensity assessment method for web questionnaires. Seismol Res Lett 86:985-990. https://doi.org/10.1785/0220140229

Tosi P, De Rubeis V, Sbarra P (2017) Frequency ranges and attenuation of macroseismic effects. Geophys J Int 210:1765-1771. https://doi.org/10.1093/gji/ggx201

Vannucci G, Tripone D, Gasperini P, Ferrari G, Lolli B (2015) Automated assessment of macroseismic intensity from written sources using the fuzzy sets. Bull Earthq Eng 13:2769-2803. https://doi. org/10.1007/s10518-015-9759-5

Van Noten K, Lecocq T, Sira C, Hinzen KG, Camelbeeck T (2017) Path and site effects deduced from merged transfrontier internet macroseismic data of two recent M4 earthquakes in northwest Europe using a grid cell approach. Solid Earth 8:453-477. https://doi.org/10.5194/se-8-453-2017

Wald DJ, Quitoriano V, Worden CB, Hopper M, Dewey JW (2012) USGS “Did you feel it?" Internet-based macroseismic intensity maps. Ann Geophys 54:688-707. https://doi.org/10.4401/ag-5354

Woith H, Petersen GM, Hainzl S, Dahm T (2018) Can animals predict earthquakes? Bull Seismol Soc Am 108:1031-1045. https://doi.org/10.1785/0120170313

Wood H, Neumann F (1931) Modified Mercalli intensity scale of 1931. Bull Seismol Soc Am 21:277-283

Publisher's Note Springer Nature remains neutral with regard to jurisdictional claims in published maps and institutional affiliations. 